\title{
Étude sur la présence des étudiants internationaux au Saguenay-Lac-St-Jean
}

\author{
Abderrahman Hassi \\ Université du Québec à Chicoutimi
}

A vec l'avènement de la révolution du monde de l'information, l'enseignement est apparu comme un facteur déterminant d'épanouissement et de prospérité pour les sociétés et, du même fait, comme une condition d'harmonie entre les pays. Une telle conviction est appuyée par la mondialisation des économies et la création de regroupements de marchés régionaux.

Il est d'ores et déjà incontournable que tout succès de tout pays passe par son adaptation au monde actuel et, par voie de conséquence, par l'accès au savoir et la participation au progrès des connaissances, en d'autres termes par la formation et le développement des ressources humaines.

Les pays développés peuvent participer à une économie basée sur la science et la technologie grâce à l'université. Néanmoins, les acquis ne pourront être maintenus et les pays moins développés ne pourront les joindre que dans la mesure où se créent des liens, des échanges, bref une mobilité des étudiants, entre autres.

L'accessibilité pour tous à la compétence, aux standards internationaux et aux marchés mondiaux passe inévitablement par une ouverture à la culture des autres, tout comme elle exige l'internationalisation de la formation, chose que l'on constate de plus en plus dans l'apprentissage des langues étrangères, les programmes d'étude à dimension internationale, les échanges internationaux d'étude, d'enseignement, d'experts ainsi que la coopération entre établissements étrangers en recherche et enseignement.

\section{Apports de la présence des étudiants internationaux}

Sans nul doute, la présence des étudiants internationaux sur les campus universitaires est fructueuse aussi bien pour l'université d'accueil que pour les étudiants locaux en raison de la diversité culturelle, du rayonnement institutionnel et des retombées économiques.

Une telle situation est d'autant plus enrichissante si l'université encourage les activités sociales, académiques et associatives tout en favorisant les rencontres entre étudiants de toutes nationalités et en leur offrant une tribune afin de partager leurs expériences internationales avec les autres. Ils développent chez leurs homologues locaux l'ouverture sur le monde et leur permettent d'acquérir des connaissances internationales et des compétences interculturelles.

$\mathrm{Au}$ plan de la contribution intellectuelle et académique, les étudiants internationaux œuvrent pour l'avancement des connaissances, tout en assurant un lien avec les établissements à l'étranger en vue de créer des coopérations ou partenariats. Le choc culturel, quant à lui, représente une source d'innovation. Certains spécialistes considèrent la présence d'étudiants internationaux comme un indice de renommée internationale de l'université.

L'apport économique de la présence des étudiants internationaux n'est pas négligeable non plus. Ils contribuent à l'économie du pays d'accueil tout au long de leur séjour. Après leur retour, éventuellement, ils favorisent des partenariats économiques, surtout 
ceux qui accèdent à des postes de décision. Les étudiants internationaux injectent chaque année plus de 11 milliards de dollars dans l'économie américaine, selon le rapport Open Doors ${ }^{1}$. En 1998, le gouvernement de la Nouvelle-Zélande souhaitait attirer 3000 étudiants chinois du fait que ceux-ci généreraient 30 milliards de dollars néo-zélandais ${ }^{2}$. D'après une étude réalisée au Royaume-Uni en 1994, on a constaté que les étudiants internationaux ont dépensé plus de 716 millions de livres sterling pour l'année 1992-1993 en matière de frais de scolarité et en biens et services produits au Royaume-Uni. Selon une enquête du Bureau canadien de l'éducation internationale (BCEI), chaque étudiant international inscrit à l'université en 1998-1999 dépensait en moyenne à peu près $18120 \$$ annuellement. Le ministère des Affaires étrangères et du Commerce international estime que les étudiants internationaux ont contribué pour 2,7 milliards de dollars à l'économie canadienne, dont 700 millions de dollars pour le Québec en 1996, ce qui équivaut à 21000 emplois $^{3}$. Il s'ensuit donc que la présence des étudiants internationaux, sur les campus universitaires, est fructueuse, que ce soit sur le plan culturel, académique ou économique.

Alors, un vaste questionnement émerge : où se positionne l'Université du Québec à Chicoutimi (UQAC) et la région du Saguenay-Lac-Saint-Jean par rapport à la mobilité des étudiants internationaux ?

\section{Objectifs de l'étude}

Selon les tendances démographiques, il y aurait une décroissance de la population régionale dans les prochaines années, ce qui pourrait avoir un impact négatif sur la clientèle de l'Université et son financement. De même, il y a une tendance de plus en plus accrue d'étudiants internationaux qui s'inscrivent auprès des universités de l'extérieur, particulièrement au Québec.

Avec la mondialisation, les entreprises de la région auront besoin de ressources humaines étrangères pour tirer un meilleur parti de leurs transactions avec l'extérieur. De surcroît, on augmente les ressources multiculturelles au Québec à travers la mondialisation de la formation.

Considérant ce contexte, l'étude s'est proposée d'élaborer des stratégies relatives au recrutement, à l'accueil et à l'intégration des étudiants internationaux tant au Département des sciences économiques et administratives de l'UQAC que dans la région du Saguenay-Lac-Saint-Jean, dans le dessein d'augmenter l'effectif de cette catégorie d'étudiants et de faciliter leur intégration aussi bien académique que sociale.

\section{En définitive, I'UQAC est par excellence le milieu qui reflète le plus un visage multiculturel au Saguenay-Lac-Saint- Jean, tout spécialement en matière de diversité (52 pays).}

Le point de départ de l'étude est constitué par une multitude d'interrogations, motivées fondamentalement par le souci de susciter des propositions d'actions et d'initiatives sur un thème majeur nécessitant un moment d'analyse et de réflexion.

Le but était donc d'examiner le processus de recrutement, d'accueil et d'intégration des étudiants internationaux à l'Université et dans la région du SaguenayLac-Saint-Jean. Cependant, avant de s'y attarder, nous avons brossé le profil de la mobilité académique internationale qui s'est transformée en véritables migrations, souvent transcontinentales.

\section{Mobilité internationale des étudiants}

Nous avons constaté que la catégorie des résidents permanents croît plus vite que la catégorie des visas d'études, ce qui amène à souligner l'impact de la politique d'immigration (la procédure qui consiste à faire venir des immigrants reçus pour une installation définitive et permanente) plutôt qu'exclusivement celle du recrutement accomplie par les universités.

À l'échelle du Québec et de l'Université du Québec, la croissance des inscriptions internationales en administration est plus rapide que celle de toutes les disciplines ${ }^{4}$. À l'UQAC, cette évolution se fait en sens contraire. En définitive, l'UQAC est par excellence le milieu qui reflète le plus un visage multiculturel au Saguenay-Lac-Saint-Jean, tout spécialement en matière de diversité (52 pays). Depuis sa création, cette institution représente le pôle majeur d'attraction et de rétention de l'immigration dans la région. Seulement 1980 immigrants vivaient dans la région en 2001, selon la Direction de la population et de la recherche du ministère des Relations avec les citoyens et de 
l'Immigration (MRCI). Quant à l'effectif des étudiants internationaux à l'UQAC, sa répartition est présentée au tableau 1.

\section{La concurrence}

L'étude de la compétitivité (universités francophones du Québec) a permis de constater que toutes les universités concurrentes ont enregistré une croissance, sauf l'Université du Québec à Rimouski, durant la période 2000-2003. Laquelle croissance trouve son explication au niveau de la catégorie des résidents permanents, tandis que la catégorie des études avec visa a évolué moins vite, voire a accusé des baisses dans certains cas.

En général, les universités situées dans des centres urbains ont évolué plus rapidement que celles situées dans les régions parce qu'elles ont vraisemblablement bénéficié des effets de la politique d'immigration qui attire la majorité des résidents permanents. Si les politiques d'immigration ne favorisent pas les régions, on s'attend à ce que les universités des centres urbains continuent d'accroître leur effectif international.

\section{Tableau 1 - Évolution du nombre d'étudiants internationaux en administration à l'UQAC par rapport au total international et à l'effectif global}

\begin{tabular}{|c|c|c|c|c|c|}
\hline Année & $\begin{array}{c}\text { Total } \\
\text { international en } \\
\text { administration }\end{array}$ & $\begin{array}{c}\text { Total } \\
\text { international } \\
\text { toutes disciplines }\end{array}$ & $\begin{array}{l}\% \text { inter. admin. } \\
\text { vs total toutes } \\
\text { disciplines }\end{array}$ & Effectif global & $\begin{array}{c}\% \text { total inter. } \\
\text { toutes disciplines } \\
\text { vs effectif global }\end{array}$ \\
\hline 2000 & 20 & 141 & $14,2 \%$ & 6701 & $2,1 \%$ \\
\hline 2001 & 18 & 148 & $12,2 \%$ & 6352 & $2,3 \%$ \\
\hline 2002 & 25 & 175 & $14,3 \%$ & 6409 & $2,7 \%$ \\
\hline $\begin{array}{l}\text { Évolution } \\
2000-2003\end{array}$ & $45 \%$ & $52,5 \%$ & - & $-3,4 \%$ & - \\
\hline
\end{tabular}

Source : MELS, Fichiers GDEU, D515 J. La Haye, $1^{\mathrm{er}}$ avril 2005.

\section{Enquêtes et constats}

Comme le mandat de l'étude était d'élaborer un programme de recrutement, d'accueil et d'intégration des étudiants internationaux au Département des sciences économiques et administratives de l'UQAC, deux enquêtes ont été menées: l'une qualitative et l'autre quantitative.

L'étude qualitative a permis de colliger les propos de quatorze personnes appartenant aux différents services de l'UQAC. Leur caractéristique commune est l'intervention dans le processus en question. Nous avons constaté que le recrutement en est à ses débuts à l'UQAC et que l'intégration se déroule de façon structurée. De nombreuses activités sont organisées aussi bien par les Services aux étudiants que par les diverses associations. À part la délocalisation des programmes, le département entreprend d'autres démarches en concertation avec l'UQAC. Les informateurs ont suggéré une panoplie de solutions et ils sont una- nimement de l'avis que la présence des étudiants internationaux tant à l'Université que dans la région est une expérience enrichissante. Il s'agit là d'un constat très net qui a émergé de tous les renseignements recueillis.

L'étude quantitative s'est déroulée au moyen de questionnaires destinés aux étudiants internationaux, professeurs du département, étudiants québécois et dirigeants d'entreprises de la région. L'objectif était de sonder leurs perceptions vis-à-vis de la présence de cette catégorie d'étudiants.

La clientèle internationale entend parler de l'UQAC par des amis (40\%) et via Internet $(23 \%)$. Une part de $35 \%$ des étudiants internationaux ayant participé à l'étude ont choisi l'UQAC en raison d'une entente avec leur institution, $29 \%$ en raison du programme d'étude et $29 \%$ ont été référés par des amis. Selon l'enquête, l'accueil des étudiants internationaux à l'UQAC est généralement bon. Les personnes inter- 
viewées rencontrent quelques problèmes au moment de la rédaction des travaux, quand ils parlent en public ou lors des travaux en équipe. Leur niveau de satisfaction du programme est moyen (65\% des répondants). Au plan de l'intégration professionnelle, la majorité mentionne la difficulté de trouver un stage dans la région $(74 \%)$ et le fait que la possibilité de trouver un emploi dans la région est faible (90\%). Seulement $6 \%$ des répondants pensent rester dans la région après la fin de leurs études. L'étroitesse du marché est de loin le facteur qui explique leur départ.

Quant aux professeurs du département, la majorité a accumulé une expertise avec les étudiants internationaux. En effet, $65 \%$ travaillent avec eux depuis plus de trois ans. Une part de $47,6 \%$ des répondants ont affirmé qu'ils participent au recrutement des étudiants internationaux. La moitié a déclaré avoir eu recours à des mesures particulières pour faciliter l'intégration académique des sujets. Les répondants estiment que la condition de réussite la plus déterminante est fondamentalement la connaissance de la langue française. Cependant, la moitié juge que la chance de réussite professionnelle des étudiants internationaux dans la région est faible.

\section{De toute évidence, la mise en œuvre des plans d'action préconisés et des stratégies va générer des profits pour l'université.}

Les étudiants québécois interrogés pensent que les compétences linguistiques représentent le point qui est le plus sujet à amélioration. Le tiers des répondants a accepté de jouer un rôle de mentor.

La consultation des entreprises régionales a souffert de représentativité en raison du taux de réponse qui est moins élevé, soit $12 \%$. N'empêche qu'il en ressort certaines données pertinentes. Plus de la moitié des répondants déclarent que la compétence et la motivation de la main-d'œuvre sont essentiellement les besoins les plus importants des entreprises de la région. La moitié des répondants est fortement en accord que l'intégration des étudiants internationaux dans le milieu de travail, par le biais des stages, est souhaitable pour les entreprises; ils déclarent qu'ils pourraient possiblement recevoir des étudiants internationaux comme stagiaires. Une proportion de
$33,3 \%$ a indiqué que la motivation au travail est la qualité qui a été la plus appréciée chez les stagiaires, tandis que $60 \%$ ont trouvé des lacunes au plan de la maîtrise du français chez les sujets. Les compétences professionnelles représentent le critère de sélection le plus déterminant dans le processus d'embauche. Néanmoins, aucun répondant ne s'attend à ce qu'un étudiant international apporte des services linguistiques à son entreprise. Par ailleurs, 43,7\% des personnes interviewées ont évoqué une vision différente comme contribution de ces étudiants. Dans le dessein de faciliter l'intégration professionnelle des étudiants internationaux dans les entreprises régionales, 38,5\% des personnes interrogées ont suggéré le mentorat. Aucun répondant n'a écarté la possibilité d'embaucher des étudiants internationaux finissants de 1'Université du Québec à Chicoutimi.

Somme toute, l'étude a abouti aux recommandations suivantes (en ce qui a trait au Département des sciences économiques et administratives seulement) :

- recruter douze étudiants internationaux par année, soit soixante nouveaux étudiants dans cinq ans;

- ajouter deux pays de provenance par année, soit dix nouveaux pays dans cinq ans;

- améliorer l'accueil des étudiants internationaux de façon à faciliter leur intégration et améliorer l'image de l'Université en vue de favoriser le recrutement;

- offrir cinq stages par année aux étudiants internationaux;

- favoriser l'embauche de deux étudiants internationaux par année, soit dix dans cinq ans.

L'étude a également permis d'élaborer des stratégies de recrutement, d'accueil et d'intégration des étudiants internationaux au département en se fondant sur un ensemble de thèmes.

De toute évidence, la mise en œuvre des plans d'action préconisés et des stratégies va générer des profits pour l'université. D'autres impacts de cette présence seraient incommensurables, et pour l'Université et pour la région.

L'intérêt de la recherche était de réfléchir au bien-être des étudiants internationaux au moyen d'un bon accueil, d'une meilleure intégration académique et so- 
cioprofessionnelle. Une telle action permettra d'en recruter davantage dans le dessein de compenser la baisse démographique dans la région et, du même coup, offrir aux entreprises régionales des ressources humaines multiculturelles en vue de faire face aux défis de la mondialisation et de la conquête de nouveaux marchés.

Il y a lieu de noter que l'apport de cette étude réside au plan de sa vision globale. En effet, nous avons consulté divers intervenants : fonctionnaires de l'Université, étudiants internationaux, étudiants québécois, professeurs du département, exécutifs d'association et entrepreneurs de la région. En outre, le recensement des écrits n'a fait état d'aucune étude ayant exclusivement traité de ce sujet auparavant.

En matière de difficultés, limites et insuffisances, nous sommes conscient des enjeux de la recherche. En fait, les informations recueillies sur la compétitivité (stratégies commerciales, façons de faire, prévisions...) ont été puisées depuis les sites Internet des universités concurrentes. Par voie de conséquence, il peut s'agir d'informations promotionnelles pour attirer la clientèle. D'où la nécessité d'être vigilant. De surcroît, nous avons pris note d'une différence au niveau de la définition de l'étudiant international. Certaines sources y incluent les résidents permanents, tandis que d'autres prennent en compte les étudiants inscrits dans le cadre de la délocalisation, abstraction faite du lieu d'études. Une telle façon de procéder peut fausser les données et, subséquemment, altérer l'analyse et l'interprétation. Pour les fins de cette étu- de, nous avons considéré la première acception pour toutes les universités.

\section{En guise de conclusion}

Comme prolongement de cette étude, il est souhaitable de poursuivre un effort de réflexion et d'analyse en matière de présence des étudiants internationaux dans les entreprises de la région. Nous l'avons abordé, mais de façon brève.

La rétention des finissants de l'UQAC dans la région représente une question qui demeure en suspens et mérite d'être étudiée du fait qu'il n'existe pas suffisamment de données qui s'y rattachent.

Nous espérons que la matière présentée apportera un nouvel éclairage aux discussions engagées sur la présence des étudiants internationaux au sein de l'UQAC et dans la région.

\section{Notes et références}

1 Witherell, S. et D. Gardner (2004), « Open Doors 2004 : International Students in the U.S. »

[En ligne] http://opendoors.iienetwork.org/?p=50137.

2 Idem.

3 Idem.

4 La Haye (avril 2005), Statistiques du ministère de l'Éducation, du Sport et du Loisir du Québec (MELS), fichiers GDEU, D515 J. 


\section{Publicité}

CEE 